\title{
Overexpression of the erythropoietin receptor in RAMA 37 breast cancer cells alters cell growth and sensitivity to tamoxifen
}

\author{
LENKA ILKOVIČOVÁ ${ }^{1 *}$, NINA TROŠT $\mathrm{T}^{2,3^{*}}$, ERIKA SZENTPÉTERIOVÁ ${ }^{1}$, PETER SOLÁR $^{1}$, \\ RADOVAN KOMEL ${ }^{3}$ and NATAŠA DEBELJAK ${ }^{3}$ \\ ${ }^{1}$ Department of Cell Biology, Institute of Biology and Ecology, Faculty of Science, P.J. Šafárik University \\ in Košice, 04001 Košice, Slovakia; ${ }^{2}$ Immunology Frontier Research Center (IFReC), Osaka University, \\ Suita, Osaka 565-0871, Japan; ${ }^{3}$ Medical Centre for Molecular Biology, Institute of Biochemistry, \\ Faculty of Medicine, University of Ljubljana, SI-1000 Ljubljana, Slovenia
}

Received February 1, 2017; Accepted June 2, 2017

DOI: 10.3892/ijo.2017.4061

\begin{abstract}
Erythropoietin (EPO) is the main regulator of erythropoiesis, and its receptor (EPOR) is expressed in various tissues, including tumors. Expression of EPOR in breast cancer tissue has been shown to correlate with expression of the estrogen receptor (ER). However, EPOR promotes proliferation in an EPO-independent manner. In patients with breast cancer, EPOR is associated with impaired tamoxifen response in ER-positive tumors, but not in ER-negative tumors. Furthermore, a positive correlation between EPOR/ER status and increased local cancer recurrence has been demonstrated, and EPOR expression is associated with G-protein coupled ER (GPER). Herein, we assessed the effects of EPOR on cell physiology and tamoxifen response in the absence of EPO stimulation using two cell lines that differ only in their EPOR expression status: RAMA 37 cells (low EPOR expression) and RAMA 37-28 cells (high EPOR expression). Alterations in cell growth, morphology, response to tamoxifen cytotoxicity, and EPOR-activated signal transduction were observed. RAMA 37 cells showed higher proliferation capacity without tamoxifen treatment, while RAMA 37-28 cells were more resistant to tamoxifen and proliferated more rapidly in the presence of tamoxifen. EPOR overexpression induced cell-morphology changes upon tamoxifen treatment, which resulted in the production of cell protrusions and subsequent cell death. Shortterm treatment with tamoxifen $(6 \mathrm{~h})$ prompted RAMA 37 cells to acquired longer protrusions than RAMA 37-28 cells, which indicated a pre-apoptotic stage. Furthermore, prolonged
\end{abstract}

Correspondence to: Professor Nataša Debeljak, Medical Centre for Molecular Biology, Institute of Biochemistry, Faculty of Medicine, University of Ljubljana, Vrazov trg 2, SI-1000 Ljubljana, Slovenia E-mail: natasa.debeljak@mf.uni-lj.si

*Contributed equally

Key words: erythropoietin receptor, tamoxifen resistance, altered cell growth, breast cancer, RAMA 37, RAMA 37-28 treatment with tamoxifen $(72 \mathrm{~h})$ caused a greater reduction in RAMA 37 cell numbers, which indicated a higher rate of cell death. RAMA 37-28 cells showed prolonged activation of AKT signaling. We propose sustained AKT phosphorylation in EPOR-overexpressing cells as a mechanism that can lead to EPOR-induced tamoxifen resistance.

\section{Introduction}

The erythropoietin receptor (EPOR) is a transmembrane protein from the type I cytokine receptor superfamily (1). The majority of EPOR is expressed on erythropoietic progenitor cells in the hematopoietic tissue of the bone marrow (reviewed in ref. 2). After erythropoietin (EPO) binding, the preformed EPOR is activated and triggers several downstream signaling pathways, including those of Janus kinase 2/signal transducer and activator of transcription 5 (JAK2/STAT5), phosphatidylinositol 3-kinase/protein kinase B (PI3K/AKT), guanine nucleotide binding protein (3)/mitogen-activated protein kinase (MAPK), and protein kinase C (PKC) (reviewed in ref. 4). The activation of this signaling not only results in erythroid proliferation and differentiation, but also in protection of erythroid progenitors against apoptosis (5).

Subsequently, the presence of functional EPOR was confirmed in a number of non-hematopoietic cells, including neurons (6), endothelial cells (7), kidney cells (8), and myoblasts (9), as well as in various tumor cells. Reports have emerged over the last 6 years that have described active EPOR signaling in head and neck squamous cell carcinomas (10), cervical cancer cells (11), glioma cells (12), renal carcinoma cells (13), and breast cancer cells (14). These findings of functional EPOR for numerous body tissues then led to the description of novel physiological effects of EPO/EPOR signaling, in addition to the regulation of erythropoiesis (15). The presence of EPOR in tumor tissues and cell lines raises the question of possible adverse effects on tumor-cell proliferation and growth, and on inhibition of apoptosis. These effects might be induced by recombinant human EPO or its analogs (e.g., erythropoiesis-stimulating agents) that are used in the treatment of patients with cancers who suffer from chemotherapy-induced anemia (reviewed in ref. 16). 
Breast cancer is the second most frequent type of cancer worldwide, with over 1.5 million new cases per year. Only lung cancer accounts for more cancer deaths in women. Breast cancer cells can express a variety of growth factor receptors, which determine the molecular classification of the disease (17). The most commonly identified of these growth factor receptors is the estrogen receptor (ER) (18), which can provide important prognostic and predictive information of tumor responses to endocrine-based therapies (19). The standard therapies for ER-positive $\left(\mathrm{ER}^{+}\right)$breast cancer include the administration of selective ER-activity modulators or inhibitors of estrogen biosynthesis.

Treatment with the chemopreventive agent and selective ER-activity modulator tamoxifen was the first targeted therapy for mammary adenocarcinomas (20). Tamoxifen was approved by the US Food and Drug Administration in 1998 for the reduction of breast cancer risk in premenopausal and postmenopausal women in the USA. Tamoxifen and some other selective ER-activity modulators can have estrogen-like agonistic effects as well as anti-estrogen-like antagonistic effects that are tissue-selective or cell-type-selective. In mammary adenocarcinoma cells, tamoxifen prevents estrogen binding to the ER, which thus reduces cell proliferation and tumor growth (21). Tamoxifen has also been shown to induce apoptosis and G1 cell cycle arrest in human breast cancer cells, and to inhibit ER-independent and MAPK-induced cell proliferation (22-24).

However, some $\mathrm{ER}^{+}$breast tumors fail to respond to tamoxifen, or develop resistance with prolonged tamoxifen treatment (25). Tamoxifen resistance is rarely caused by mutations in ER (26). The alterations in signaling pathways that have been implicated in acquired tamoxifen resistance are those that affect cell survival and proliferation, the cell cycle, apoptosis, and epithelial-to-mesenchymal transition of tumor cells $(27,28)$. In this regard, tamoxifen-resistant breast cancer phenotypes are promoted by changes in AKT activity (29).

Responses of breast cancer cells to tamoxifen have also been correlated to EPOR expression. Larsson et al (30) indicated that high EPOR expression is a negative prognostic factor for recurrence-free survival of tamoxifen-treated patients with $\mathrm{ER}^{+} /$progesterone receptor $(\mathrm{PR})^{+}$breast tumors. On the contrary, recurrence-free survival of non-treated $\mathrm{ER}^{+} / \mathrm{EPOR}^{+}$breast cancer patients was significantly improved. Their study also revealed associations between EPOR, ER and PR in breast cancer cells and patients, while Volgger et al (32) further showed positive association between EPOR/ER/PR status and increased local cancer recurrence.

In terms of breast cancer cell lines, EPOR expression has been associated with expression of G-protein-coupled ER (GPER) (33). Silencing of EPOR expression via EPOR knock-down resulted in decreased proliferation of cultured $\mathrm{EPOR}^{+} / \mathrm{ER}^{+}{ }^{+}$breast cancer cells, but not of $\mathrm{ER} \alpha^{-}$cells . Recombinant human EPO stimulation of cultured cells also had no effects on cell proliferation (34).

The objective of the present study was to assess the effects of EPOR on the growth of breast cancer cells, and on their sensitivity to tamoxifen, in the absence of stimulation with recombinant human EPO. For this purpose, we compared two rat mammary epithelial cell lines that show different levels of EPOR expression. RAMA 37 cells are a benign non-invasive cell line that shows low EPOR expression, while a RAMA 37 clone, known as RAMA 37-28 cells, stably expresses higher levels of human EPOR (35). Herein, both of these cell lines were characterized for EPOR and ER expression and compared to each other in terms of their proliferation capacity, response to tamoxifen-induced cytotoxicity, and activation of protein signaling.

\section{Materials and methods}

Cell lines and cell culture. The parental non-metastatic benign tumor-derived rat mammary epithelial cell line, RAMA 37 cells, and its derived stably transformed cell subclone, RAMA 37-28 cells, were used as the model system. Transfection was carried out with the pcDNA3.1 expression vector that contained wild-type human EPOR, followed by selection of modified cells with $1.0 \mathrm{mg} / \mathrm{ml}$ geneticin (35). The cells were cultured in Dulbecco's modified Eagle's medium supplemented with high glucose $(4.5 \mathrm{~g} / \mathrm{l})$, L-glutamine (GE Healthcare, Little Chalfont, UK) and $10 \%$ fetal bovine serum (Gibco; Thermo Fisher Scientific, Inc., Waltham, MA, USA). The cell cultures were maintained at $37^{\circ} \mathrm{C}$ in a humidified $5 \% \mathrm{CO}_{2}(\mathrm{v} / \mathrm{v})$ atmosphere. Cell numbers were determined using a Coulter counter (model ZF; Coulter Electronics, Luton, UK), and total cell viability was analyzed by staining with $0.15 \%$ eosin, followed by light microscopy.

\section{Gene expression analysis}

RNA isolation. Total RNA was extracted using the TRI reagent (Sigma-Aldrich, St. Louis, MO, USA), and treated with DNase I (Roche Diagnostic, Basel, Switzerland), according to the manufacturer instructions. The quality of the RNA samples was determined using a bioanalyzer (Agilent), to assure that all RIN values were $>9.8$. Total RNA $(1 \mu \mathrm{g})$ was transcribed to cDNA using SuperScript III reverse transcriptase (Invitrogen, Carlsbad, CA, USA), according to the manufacturer's instructions.

Quantitative real-time reverse transcriptase-PCR. The PrimerExpress software (Applied Biosystems, Foster City, CA, USA) was used for the design of forward and reverse primers for EPO, EPOR, ESR1, ESR2, GPER, RPLPO and $C Y C A$ that spanned intron-exon junctions. These were checked for their specificities with the BLAST algorithm. RPLPO and $C Y C A$ were selected as the reference genes, and were used for normalization of the expression levels for the genes of interest. Quantitative real-time reverse transcriptase was performed using SybrGreen chemistry (Roche Diagnostic) on a 384-well platform of a LightCycler 480 Real-time PCR System (Roche Diagnostic). Amplification of the specific PCR products was performed in triplicate in a total reaction mixture of $5 \mu 1$, which contained $0.75 \mu \mathrm{l}$ of the cDNA template. Gene expression normalization factors were calculated using the GeNorm algorithm for each sample, based on the geometric means of the selected reference genes (31). The minimum information for publication of quantitative real-time PCR experiments (MIQE) was followed in the performance and interpretation of the qPCR reactions (36). The data are presented as relative expression levels for each gene of interest $\pm \mathrm{SD}$, from three biological replicates. 
Table I. Primary and secondary antibodies used in the western blotting.

\begin{tabular}{|c|c|c|c|c|c|}
\hline Type & Antibody & Specificity & Dilution & Manufacturer & Cat. no. \\
\hline \multirow[t]{13}{*}{ Primary } & Anti-p44/42 MAP kinase (ERK1/2) & Rabbit & $1: 1000$ & Cell Signaling Technology & 9102 \\
\hline & Anti-phospho-p44/42 & Rabbit & $1: 1000$ & Cell Signaling Technology & 9101 \\
\hline & MAP kinase (pERK1/2) & & & & \\
\hline & Anti-AKT & Rabbit & $1: 1000$ & Cell Signaling Technology & 9272 \\
\hline & Anti-phospho-AKT & Rabbit & $1: 1000$ & Cell Signaling Technology & 9271 \\
\hline & Anti-STAT5 & Rabbit & $1: 2000$ & R\&D Systems & AF2168 \\
\hline & Anti-phospho-STAT5 (Y694/Y699) & Rabbit & $1: 1000$ & R\&D Systems & AF4190 \\
\hline & Anti-EPOR (M-20) & Rabbit & $1: 400$ & Santa Cruz Biotechnology & sc697 \\
\hline & Anti-ER $\alpha(F-10)$ & Mouse & $1: 2000$ & Santa Cruz Biotechnology & sc- 8002 \\
\hline & Anti-ER $\beta(H-150)$ & Rabbit & 1:1000 & Santa Cruz Biotechnology & sc-8974 \\
\hline & Anti-GPER & Rabbit & $1: 500$ & Sigma Chemicals & HPA027052 \\
\hline & Anti- $\beta$-actin & Mouse & $1: 5000$ & Sigma Chemicals & A5441 \\
\hline & & Rabbit & $1: 2000$ & Sigma Chemicals & A2066 \\
\hline \multirow[t]{4}{*}{ Secondary } & Anti-mouse IgG & Rabbit & $1: 2000$ & Pierce & 31452 \\
\hline & & Goat & $1: 5000$ & $\begin{array}{l}\text { Jackson ImmunoResearch } \\
\text { Laboratories }\end{array}$ & $115-035-068$ \\
\hline & Anti-rabbit IgG & Goat & $1: 2000$ & Pierce & 31461 \\
\hline & & Goat & $1: 5000$ & $\begin{array}{l}\text { Jackson ImmunoResearch } \\
\text { Laboratories }\end{array}$ & $111-035-003$ \\
\hline
\end{tabular}

\section{Cell proliferation}

MTT assay. The cells $\left(12.5 \times 10^{3}\right)$ in the medium supplemented with $1 \%$ or $10 \%$ fetal bovine serum were seeded as quintuplicates in 96-well plates, and left to adhere for $24 \mathrm{~h}$. Cell proliferation was determined using 3-(4.5-dimethylthiazol2-yl)-2.5-diphenyl tetrazolium bromide (MTT reagent; Sigma-Aldrich), and was measured for five sequential days. Each day, MTT reagent was added to the wells of one plate, which was then incubated for $4 \mathrm{~h}$ under the standard culture conditions. Viable cells with active metabolism can convert MTT into an insoluble formazan product, which can be dissolved by addition of $20 \%$ sodium dodecyl sulfate (in $\mathrm{HCl}$ ). Absorbance was measured at $570 \mathrm{~nm}$ using a spectrophotometer (Epoch; BioTek, Winooski, VT, USA). After the blank subtraction, the fold-changes in cell proliferation were determined for each day, by normalization to the proliferation levels after $24 \mathrm{~h}$ of culturing. The data are presented as mean fold-changes in cell proliferation $\pm \mathrm{SD}$, of three independent experiments.

Clonogenic assays. The cells were seeded in 6-well plates in triplicate at 100 cells/well and cultured for 14 days. The culture medium was changed every 5 days. Colony quantification was carried out manually and using the UviPro software after crystal violet staining (0.5\%; Sigma-Aldrich). Colonies were classified as small if they contained $<100$ cells, and otherwise they were classified as big. The data are presented as mean colony numbers $\pm \mathrm{SD}$, of three independent experiments.

Cell viability assays

$x$ CELLigence. The experiments were performed using an xCELLigence dual-plate real-time cell analyzer (Roche
Diagnostic), which was placed in a humidified incubator at $37^{\circ} \mathrm{C}$ and $5 \% \mathrm{CO}_{2}$. Cell proliferation and cytotoxicity were determined using 16-well plates (E-plates; Roche Diagnostic) with electrodes attached to the bottom for impedance-based detection of cell attachment, spreading, and proliferation. The impedance of each well was automatically monitored using the xCELLigence system, which was expressed as the Cell Index. Initially, $100 \mu 1$ cell-free media (with $10 \%$ fetal bovine serum) was added to the wells and incubated for $30 \mathrm{~min}$ at room temperature. This was following by addition of $50 \mu 1$ cell suspension (for 1,250 cells/well). The cells were left to adhere for $30 \mathrm{~min}$ at room temperature, and then placed on the real-time cell analyzer for $72 \mathrm{~h}$. The Cell Index was monitored every hour. After the initial $72 \mathrm{~h}$ of culture, the cells were treated with tamoxifen (Sigma-Aldrich) at 25, 37, 50, 75, 100 or $150 \mu \mathrm{M}$ (in ethanol). Ethanol-treated cells and cells without tamoxifen treatment were used as controls. Each tamoxifen concentration was tested in duplicate within the same experiment, and the Cell Index was again monitored every hour, to $48 \mathrm{~h}$ of tamoxifen treatment.

Normalized Cell Index. The normalized Cell Index (37) was calculated by its normalization with the Cell Index at the point of tamoxifen treatment (i.e., $72 \mathrm{~h}$ ). The data are presented as the mean normalized Cell Index curves $\pm \mathrm{SD}$, of three independent experiments.

IncuCyte ZOOM system. Experiments were performed using an IncuCyte ZOOM system (Essen BioScience, Ann Arbor, MI, USA), which consists of a microscope gantry in a humidified incubator at $37^{\circ} \mathrm{C}$ and $5 \% \mathrm{CO}_{2}$, and a networked external controller hard drive that gathers and processes image data. 


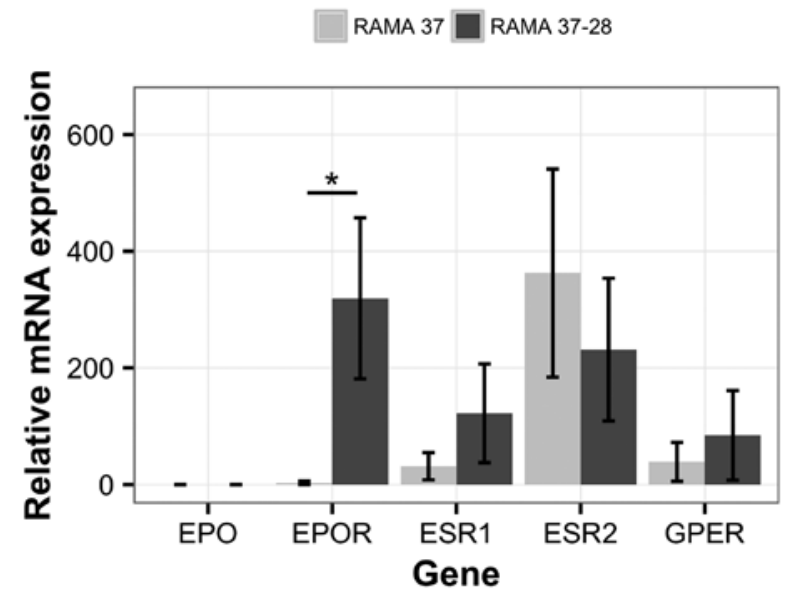

Figure 1. Gene expression of EPO, EPOR, ESR, and GPER in RAMA 37 and RAMA 37-28 cells. Data are means \pm standard deviations of three independent experiments. "Statistically significant difference of Type 1 error $\alpha<0.05$ for gene expression between RAMA 37 and RAMA 37-28 cells.

The cells were seeded in 96-well plates in sextuplicates at 2,000 cells/well (as $100 \mu \mathrm{l}$ cell suspension/well) and placed in the IncuCyte ZOOM system. After the initial $24 \mathrm{~h}$ of culture, the cells were treated with $25 \mu \mathrm{M}$ tamoxifen (dissolved in ethanol). Ethanol-treated cells and those growing in media without tamoxifen were used as controls. The IncuCyte ZOOM system automatically monitored the cell confluence in each well through a $x 20$ objective (Nikon) every $2 \mathrm{~h}$, until $72 \mathrm{~h}$ of tamoxifen treatment. The experiment was performed three times.

Western blotting. Western blotting was carried out according to standard protocols. Using the MTT assay, $50 \mu \mathrm{M}$ tamoxifen was defined as the optimal concentration for analysis of protein signaling. The cells were grown in the standard growth media for $24 \mathrm{~h}$ and then exposed to $50 \mu \mathrm{M}$ tamoxifen for $0,5,10,15,30$ and $60 \mathrm{~min}$. The cells were then lysed for $10 \mathrm{~min}$ in lysis buffer on ice, as described by Kutuk et al (38), and the soluble proteins were recovered in the supernatant following centrifugation at $12,000 \mathrm{x} \mathrm{g}$ for $10 \mathrm{~min}$. Protein samples were separated using $12 \%$ SDS-PAGE gels and equal loading confirmed by detection of $\beta$-actin. This was followed by electroblotting onto Immobilon-P transfer membranes (Millipore, Billerica, MA, USA), which were then incubated overnight with the primary antibodies, as specified in Table I. The membranes were then incubated with the secondary horseradish-peroxidase-conjugated antibody (Table I) for $1 \mathrm{~h}$, and the antibody reactivity was visualized using ECL Western blotting substrate (Pierce, Waltham, MA, USA) and Kodak Biomax film (Sigma-Aldrich). The data are shown as a representative figure and in Table as means of the densities recorded from three independent experiments.

Statistical analysis. The significance of the differences in gene expression and colony formation capacity between the RAMA 37 and RAMA 37-28 cells were determined using Student's t-tests. Statistical significance for cell proliferation or in response to tamoxifen-induced cytotoxicity was determined using two-way ANOVA tests, while one-way ANOVA

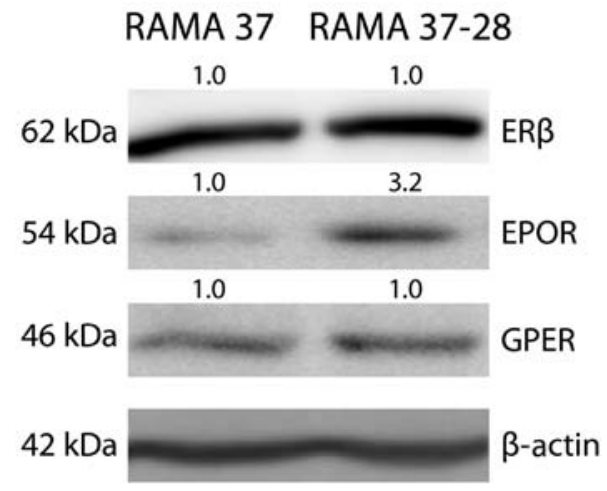

Figure 2. Representative protein levels of $E R \beta$ (the protein product of $E S R 2$ ), EPOR and GPER in RAMA 37 and RAMA 37-28 cells. Equal loading was confirmed by detection of $\beta$-actin. Ratios indicated represent quantitative analysis of the densitometric values of the specific band intensities normalized to RAMA 37 cells, which were arbitrarily set to 1 .

was used to assess the statistical differences in the protein signaling. $\mathrm{P}<0.05, \mathrm{P}<0.01$ or $\mathrm{P}<0.001$ were considered as statistically significant.

\section{Results}

Expression of erythropoietin and estrogen receptor. Real-time PCR analysis of EPO, EPOR, ESR1, ESR2, and GPER was performed to determine the level of endogenous $E P O$ mRNA and the differences in receptor status between the RAMA 37 and RAMA 37-28 cells (Fig. 1). Endogenous EPO expression was not confirmed in the tested cell lines. EPOR mRNA levels were not detectable in the parental RAMA 37 cells, while relatively high expression was seen for the RAMA 37-28 cells, which had been stably transfected with human EPOR (35). ESR1 expression was low in both cell lines, while ESR2 was slightly higher in RAMA 37 cells. However, the difference in $E S R 2$ expression between the two cell lines was not statistically significant. The expression level of GPER was low in both cell lines.

The EPO, EPOR, and ER expression was evaluated at the protein level. The expression of ER $\beta$ (i.e., the protein product of ESR2), EPOR, and GPER was confirmed in both cell lines (Fig. 2). Low levels of EPOR were detected in the parental RAMA 37 cells, with higher expression in the RAMA 37-28 cells. EPO and ER $\alpha$ (i.e., the protein product of ESR1) were not detected at the protein level.

Effects of EPOR overexpression on cell growth. The RAMA 37 and RAMA 37-28 cells were compared in terms of cell growth and proliferation, and their clonogenic characteristics, to evaluate the effects of EPOR overexpression on the cell physiology (Figs. 3 and 4). Over 5 days, RAMA 37 cells showed higher proliferation levels compared to RAMA 37-28 cells in media supplemented with $1 \%$ or $10 \%$ fetal bovine serum (Fig. 3). Statistically significant differences $(\mathrm{P}<0.05)$ were confirmed for days $4-5$ in $1 \%$ fetal bovine serum, and for days 3-5 in 10\% fetal bovine serum.

Colony numbers were determined according to colony size (Fig. 4). Colonies were considered small if they containing $<100$ cells, or they were considered big at $>100$ cells. The 


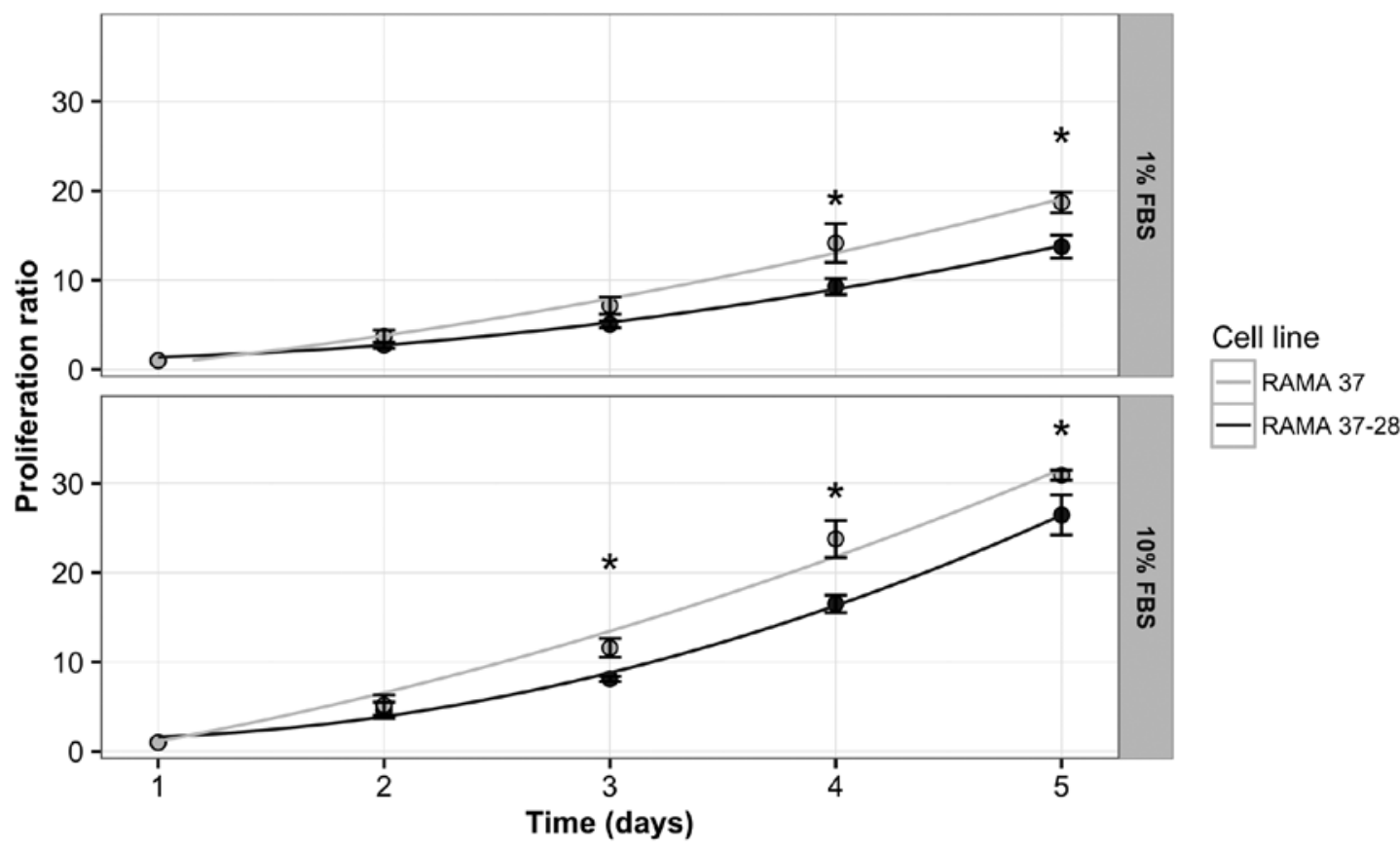

Figure 3. Proliferation rates of RAMA 37 and RAMA $37-28$ cells in growth media supplemented with 1\% (top) or 10\% (bottom) fetal bovine serum. Proliferation levels were measured over 5 consecutive days using the MTT assay. Fold-changes in cell proliferation levels were determined by normalization of mean absorbance values measured for a particular day with the mean value measured at day 1 . Data are means \pm standard deviations of three independent experiments. "Statistically significant difference of Type 1 error $\alpha<0.05$.
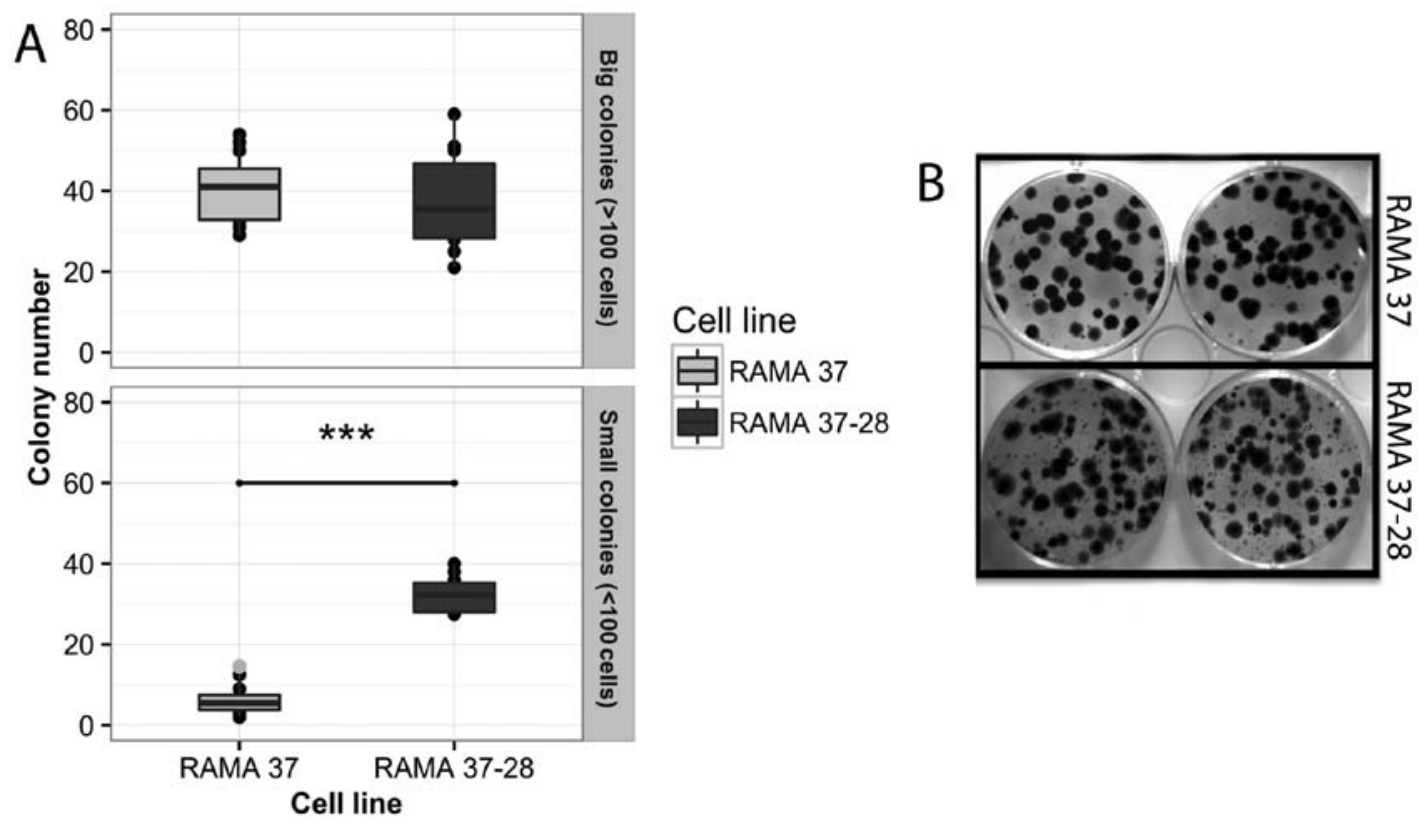

Figure 4. Colony formation assays for RAMA 37 and RAMA 37-28 cells. (A) Box plots showing the number of big colonies with $>100$ cells, and small colonies with $<100$ cells. These also present the full range of variation in the sample population (from minimum to maximum) and the likely range of variation (box), with the median. Data are means \pm standard deviations of three independent experiments. Statistically significant ${ }^{* * *} \mathrm{P}<0.001$. (B) Representative clonogenic assay for RAMA 37 and RAMA 37-28 cells.

number of big colonies was comparable for both of these cell lines. However, the number of small colonies was significantly higher for RAMA 37-28 cells.

Effects of EPOR on tamoxifen resistance. The proliferation of RAMA 37 and RAMA 37-28 cells and their responses to tamoxifen treatment were screened using the xCELLigence system (Fig. 5). The cells were plated and left to proliferate for $72 \mathrm{~h}$, and then they were treated with tamoxifen for $48 \mathrm{~h}$ (i.e., to $120 \mathrm{~h}$ ). The proliferation capacity of RAMA 37 cells under the control conditions was significantly higher that for the RAMA 37-28 cells (Fig. 5A). However, the RAMA 37-28 cells were more resistant to the tamoxifen treatment, as they showed faster proliferation in the presence of tamoxifen (Fig. 5B and C). 


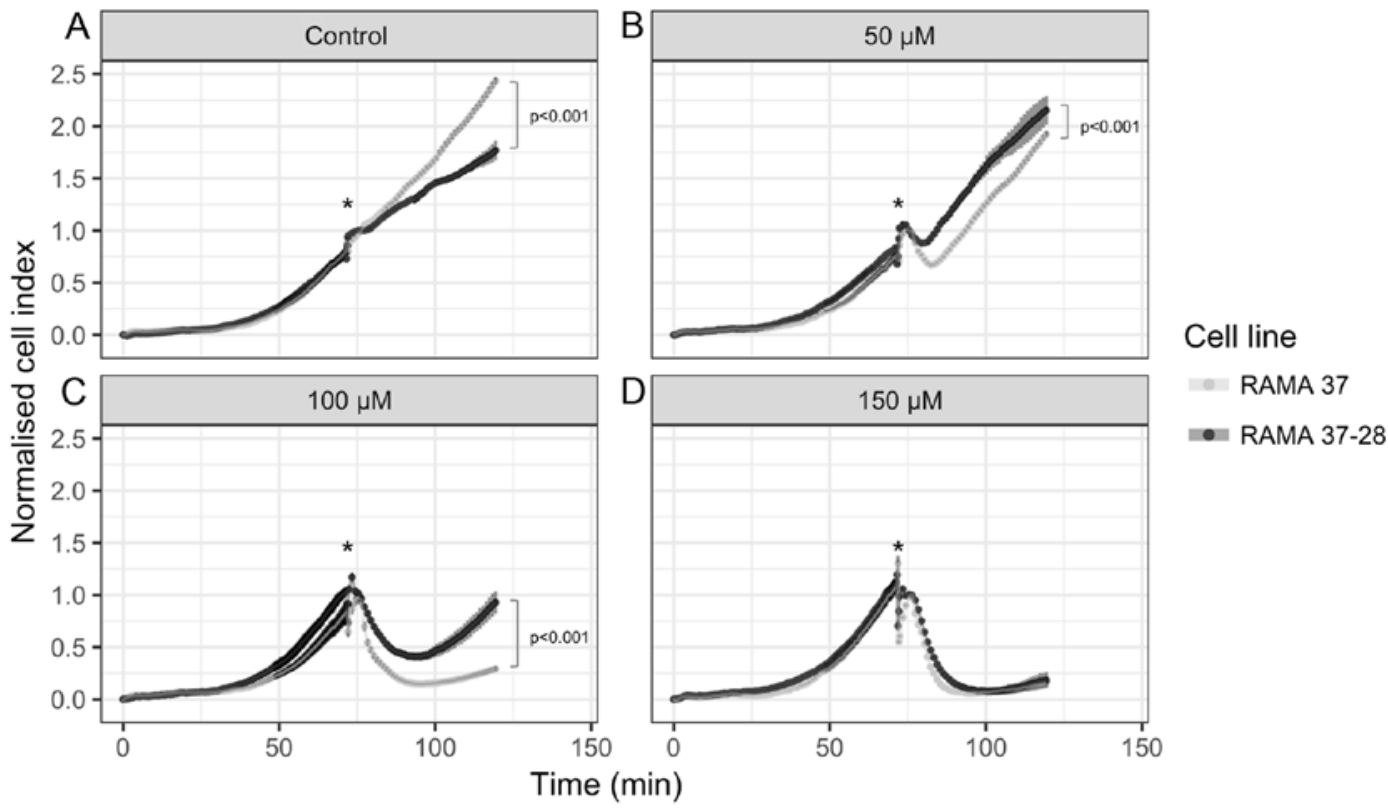

Figure 5. Effects of tamoxifen treatment on proliferation and viability of RAMA 37 and RAMA 37-28 cells. (A) Control without tamoxifen, (B) with $50 \mu \mathrm{M}$ tamoxifen treatment, (C) with $100 \mu \mathrm{M}$ tamoxifen treatment, (D) with $150 \mu \mathrm{M}$ tamoxifen treatment. The cells were left to proliferate in standard growth media for $72 \mathrm{~h}$, followed by treatment with tamoxifen for $48 \mathrm{~h}$ [indicated with asterisk $\left(^{*}\right)$ ]. Cell viability was monitored using an xCELLigence system. Data show normalized Cell Index curves \pm standard deviations of three independent experiments. The normalized Cell Index was arbitrarily set to 1 at the point of tamoxifen treatment $(72 \mathrm{~h})$. Statistically significant difference of $\mathrm{P}<0.001$ is indicated.
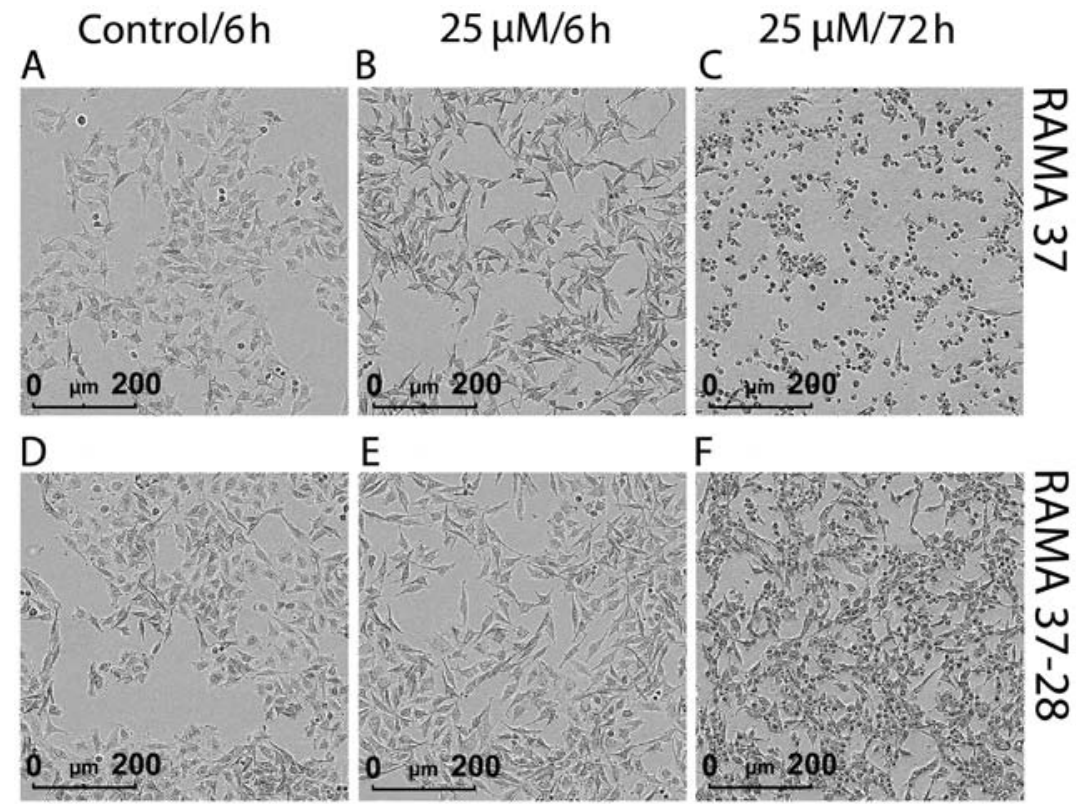

Figure 6. Effects of tamoxifen treatment on morphology and confluency of RAMA 37 and RAMA 37-28 cells. Cells were left to proliferate for $6 \mathrm{~h}$ under control conditions without tamoxifen (A and D), $6 \mathrm{~h}$ with $25 \mu \mathrm{M}$ tamoxifen treatment (B and E), and $72 \mathrm{~h}$ with $25 \mu \mathrm{M}$ tamoxifen treatment (C and F). Cell confluency was monitored using the IncuCyte ZOOM system. Scale bar: $200 \mu \mathrm{m}$.

The highest tamoxifen concentration $(150 \mu \mathrm{M})$ was lethal for both of these cell lines (Fig. 5D).

The morphological changes of the RAMA 37 and RAMA 37-28 cells upon tamoxifen treatment were assessed using the IncuCyte ZOOM system, which monitors cell proliferation by determining the area occupied by the cells (i.e., the percent confluence) in images taken over time (Fig. 6). The cells were plated and left to proliferate for $24 \mathrm{~h}$, followed by $25 \mu \mathrm{M}$ tamoxifen treatment for $72 \mathrm{~h}$. This tamoxifen treatment promoted morphological changes in both cell lines, although they were more evident and more rapid in onset for RAMA 37 cells (Fig. 6A-C). Short-term tamoxifen treatment $(6 \mathrm{~h})$ promoted the formation of nanotube-like structures (i.e., protrusions) that were more pronounced and longer for RAMA 37 cells (Fig. 6B). These morphological changes might be indicative of a pre-apoptosis stage. Moreover, prolonged treatment with tamoxifen $(72 \mathrm{~h})$ promoted greater cell death for RAMA 37 cells (Fig. 6C), with RAMA 37-28 cells not 
RAMA 37

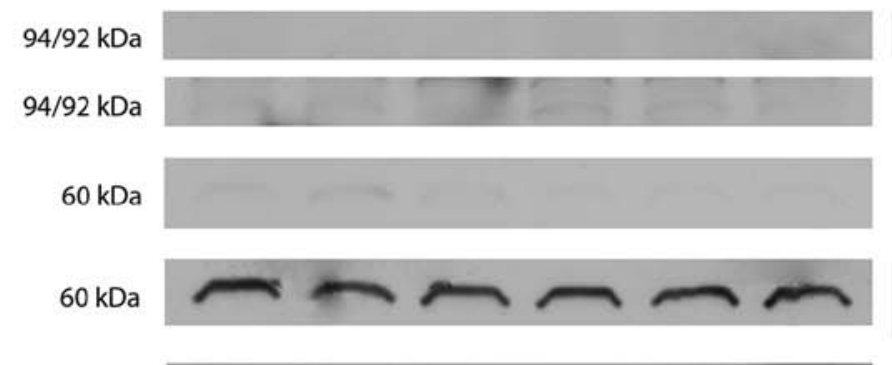

$44 / 42 \mathrm{kDa}$

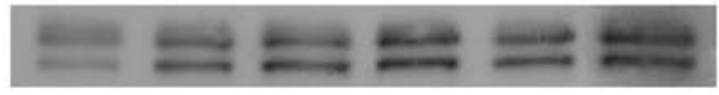

$44 / 42 \mathrm{kDa}$

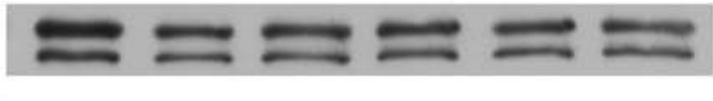

$42 \mathrm{kDa}$

$50 \mu \mathrm{M}$

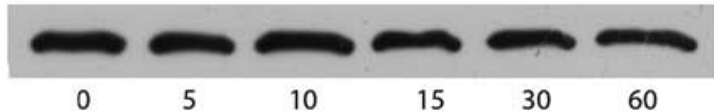

RAMA 37-28
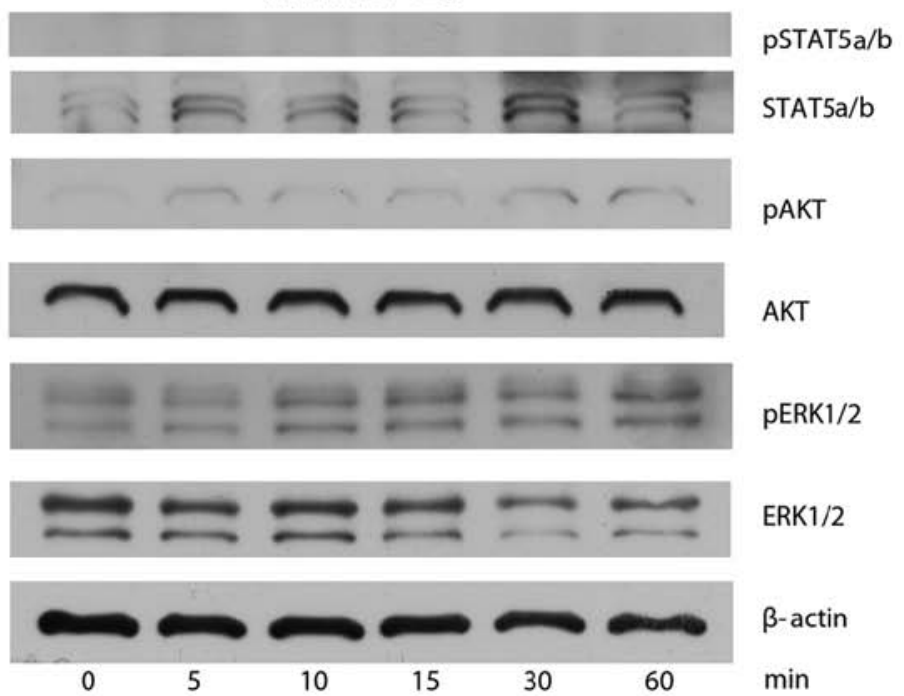

Figure 7. Effects of tamoxifen treatment on phosphorylation of STAT5a/b, AKT, and ERK1/2 in RAMA 37 and RAMA $37-28$ cells. The cells were grown in standard growth media for $24 \mathrm{~h}$ and then treated with $50 \mu \mathrm{M}$ tamoxifen for $0,5,10,15,30$ and 60 min. Equal loading was confirmed by detection of $\beta$-actin. Representative image of three independent experiments is shown.

Table II. Effect of tamoxifen treatment on AKT and ERK1/2 phosphorylation in RAMA 37 and RAMA 37-28 cells.

\begin{tabular}{llcccccc}
\hline & & \multicolumn{5}{c}{ Ratio according to tamoxifen treatment (min) } \\
\cline { 3 - 7 } Cell type & $\begin{array}{l}\text { Target } \\
\text { ratio }^{\mathrm{a}}\end{array}$ & 0 & 5 & 10 & 15 & 30 & 60 \\
\hline RAMA 37 & pAKT & $1.00 \pm 0.00$ & $1.42 \pm 0.44$ & $0.86 \pm 0.12$ & $0.53 \pm 0.02^{\mathrm{d}}$ & $0.32 \pm 0.03^{\mathrm{d}}$ & $0.30 \pm 0.06^{\mathrm{d}}$ \\
& pERK1/2 & $1.00 \pm 0.00$ & $1.97 \pm 0.27$ & $2.27 \pm 1.00$ & $2.32 \pm 0.71^{\mathrm{b}}$ & $2.24 \pm 0.34^{\mathrm{b}}$ & $2.74 \pm 0.86^{\mathrm{b}}$ \\
RAMA 37-28 & pAKT & $1.00 \pm 0.00$ & $1.38 \pm 0.30$ & $1.03 \pm 0.18$ & $0.83 \pm 0.09$ & $1.15 \pm 0.30$ & $1.16 \pm 0.30$ \\
& pERK1/2 & $1.00 \pm 0.00$ & $1.55 \pm 0.85$ & $1.40 \pm 0.27$ & $1.89 \pm 0.30^{\mathrm{b}}$ & $2.50 \pm 0.50^{\mathrm{c}}$ & $2.42 \pm 0.40^{\mathrm{c}}$ \\
\hline
\end{tabular}

${ }^{a}$ Ratios \pm standard deviation from quantitative densitometric analysis of pAKT and pERK1/2 normalized to AKT and ERK, respectively. Ratios at time 0 arbitrarily set to $1 .{ }^{b} \mathrm{P}<0.05$; ${ }^{\mathrm{c}} \mathrm{P}<0.01$; ${ }^{\mathrm{d}} \mathrm{P}<0.001$ versus time 0 (three independent experiments; one-way ANOVA tests).

affected to the same extent (Fig. 6F). Movies demonstrating the differences in the tamoxifen responsiveness of RAMA 37 and RAMA 37-28 cells are available at http://ibk.mf.uni-lj.si/ people/debeljak/RAMA.

Modified signal activation during tamoxifen treatment. The effects of tamoxifen treatment on signal transduction were determined using Western blotting for ERK1/2, AKT, and STAT5a/b phosphorylation (Fig. 7 and Table II). Upon tamoxifen treatment, no STAT5 phosphorylation (pSTAT5) was observed in RAMA 37 cells or in the EPOR-overexpressing RAMA 37-28 cells. The PI3K/AKT signaling pathway was activated already in both of these cell lines prior the tamoxifen treatment, as they both showed low levels of endogenous AKT phosphorylation (pAKT). Tamoxifen treatment then changed the AKT phosphorylation patterns in a cell-type-dependent manner. The pAKT levels were reduced in RAMA 37 cells (e.g., 15 min of tamoxifen treatment), but increased in RAMA 37-28 cells. These increased pAKT levels in RAMA 37-28 cells were still seen after 60 min of tamoxifen treatment, which suggests that the activity of the PI3K/AKT signaling pathway is increased in this cell line. Tamoxifen treatment also resulted in changes in the RAS/MAPK signaling pathway, with increased phosphorylation levels of ERK1/2 (pERK1/2) were seen. ERK1/2 phosphorylation was a little faster and stronger in RAMA 37 cells, but still comparable to that in RAMA 37-28 cells.

\section{Discussion}

Many studies have suggested that EPOR has a role in tumor progression (30) through the stimulation of cell proliferation and/or inhibition of apoptosis of cancer cells upon EPO binding $(10,14)$. In contrast, other studies have argued that although EPOR is present in cancer cells, it is not biologically active and is not essential for tumor growth $(2,39)$, as stimulation with exogenous EPO does not stimulate tumor cell proliferation (34). However, cancer cells are believed to undergo a continuous cycle of selection changes, with only the more adapted cells favored for the passing on of their genetic information. These cells are not likely to retain biologically inactive metabolic and regulatory pathways, and therefore it 
is reasonable to expect that EPOR expression in cancer cells is not redundant, but is instead involved in the tumor biology.

In the present study, we examined the effects of EPOR on the growth of breast cancer cells, and its role in the tamoxifen response. RAMA 37 and RAMA 37-28 cells differ only in their EPOR expression levels, which we confirmed with the expression analysis at the mRNA and protein levels. Indeed, there were no significant differences in the expression of ER $\alpha$ (i.e., the product of $E S R 1$ ), ER $\beta$ (i.e., the product of ESR2) or GPER between these two cell lines, indicating that they represent a good model for the evaluation of EPOR effects on cell physiology.

The ER $\alpha$ status is used as a prognostic marker in breast cancer evaluation, and it is the primary target of endocrine therapies. Generally, patients with ER $\alpha$-negative tumors cannot benefit from tamoxifen therapy, although a fraction of these tumors do appear to be sensitive to tamoxifen $(28,40)$. Furthermore, phosphorylation of $\mathrm{ER} \alpha$ by protein kinase A has been shown to convert the antagonist tamoxifen into an agonist, thereby reversing its effects on tumor cell growth. Activation of protein kinase A can occur through GPER or adenylyl cyclase, although it is difficult to achieve constitutive protein kinase A activation due to desensitization of both of these receptors upon activation (41). Additionally, low levels of ER $\beta$ are associated with tamoxifen resistance, and as such this might serve as an additional independent predictive marker (42). As we demonstrated comparable expression levels of ER $\alpha, E R \beta$, and GPER in these RAMA 37 and RAMA 37-28 cells, we propose that (an)other molecule(s) or signaling pathway(s) contribute to the tamoxifen-resistant phenotype that is observed for RAMA 37-28 cells.

We showed that EPOR overexpression in these cancer cells can influence cell proliferation and resistance even in the absence of EPO. The greater proliferation capacity of RAMA 37 cells indicates that EPOR lowered the ability of RAMA 37-28 cells to divide. When comparing the clonogenic potential of RAMA 37 and RAMA 37-28 cells, the RAMA 37-28 cells were more clonogenic. Although the numbers of big colonies ( $>100$ cells) were comparable across both of these cell lines, RAMA 37-28 cells formed a significantly greater number of small colonies ( $<100$ cells) This greater clonogenic potential of RAMA 37-28 cells has also been previously indicated (35). Reinbothe et al (34) suggested a role for EPOR in the proliferation control of ER $\alpha$ breast cancer cells, as EPOR knock-down in ER $\alpha$-positive breast cancer cell lines resulted in cell growth inhibition. Furthermore, a study on A2780 ovarian cancer cell line reported that inhibition of EPOR expression abrogated growth of its tumor xenograft (39).

Tamoxifen induces apoptosis in human breast cancer cell lines and inhibits cell proliferation in human ovarian cancer cell lines through a mechanism that is independent of overall ER expression (22-24). We observed here that while under the control conditions the proliferation potential of RAMA 37 cells was higher than that of RAMA 37-28 cells, the treatment with tamoxifen affected RAMA 37 cells more and resulted in a greater block of proliferation and a higher rate of cell death than for RAMA 37-28 cells. These data therefore suggest that EPOR can protect cells against tamoxifen-induced cell death even in the absence of EPO.
Previously, Reinbothe et al (34) showed that EPOR knock-down resulted in a more efficient tamoxifen-induced inhibition of the ER $\alpha$ activity. Herein, tamoxifen treatment caused morphological changes to RAMA 37 and RAMA 37-28 cells that corresponded to a pre-apoptotic stage. These morphology changes were manifested in the form of pronounced protrusions that were especially obvious in RAMA 37 cells, which correlated with their higher sensitivity to tamoxifen-induced cytotoxicity. Protrusions were less obvious in RAMA 37-28 cells and might correlate with the higher survival rate (greater resistance) of these RAMA 37-28 cells. Based on our knowledge, such EPOR-modulated cell morphology changes in response to tamoxifen treatment have not been described previously for breast cancer cells, and thus we will further investigate this in detail.

A correlation between signaling through AKT and tamoxifen resistance was recently shown in several studies. Shi et al (35) demonstrated the role of EPO/EPOR-induced persistent AKT activation in the transformation of RAMA 37-28 cells from a benign to a tumorigenic phenotype. Furthermore, Paragh et al (39) showed the presence of phosphorylated EPOR signaling components in A2780 human ovarian adenocarcinoma cells, even when the cells were not exposed to exogenous EPO. EPOR knock-down in breast cancer cell lines reduces pAKT levels, which suggests its involvement in transmission of signals, including phosphorylation and activation of AKT (34). Furthermore, MCF7 cell transfection with AKT showed tamoxifen resistance, as tamoxifen showed reduced inhibition of the growth of these transformed cells (43). Previous studies have also shown that simultaneous treatment of breast cancer cell lines with tamoxifen and the PI3K inhibitor, LY294002, reduced tamoxifen-induced AKT phosphorylation and significantly increased the pro-apoptotic effects of tamoxifen (29). In the present study, RAMA 37 and RAMA 37-28 cells showed similar phosphorylation status for the key EPOR signaling proteins. The tamoxifen treatment induced comparable changes in pERK1/2 for these cell lines, and had no effects on the phosphorylation of STAT5a/b. On the other hand, tamoxifen treatment caused prolonged AKT activation (pAKT) in RAMA 37-28 cells, but not in RAMA 37 cells. Given these results, we propose modified AKT signaling in RAMA 37-28 cells as the potential mechanism that leads to this increased cell resistance to tamoxifen for RAMA 37-28 cells.

In conclusion, the present study investigated the effects of EPOR expression on breast cancer cell growth and the sensitivity of these cells toward tamoxifen. The study was conducted using benign non-invasive mammary epithelial cells that express low EPOR levels, compared to the counterpart cell line with greater EPOR expression. Despite the absence of EPO in these cells, we indicated differences in cell growth, morphology, EPOR-induced signal transduction, and tamoxifen resistance across these two cell lines. The data show that tamoxifen treatment induces prolonged activation of AKT signaling in RAMA 37-28 cells but not in RAMA 37 cells. Therefore, we suggest that sustained signaling via AKT renders RAMA 37-28 cells more resistant to tamoxifen. Moreover, we report here the first evidence of EPOR-modulated cell morphology changes upon tamoxifen treatment, which result in increased formation of cell protrusions and subsequent cell death. The observed putative link between EPOR 
expression and the responses of breast cancer cells to tamoxifen might have clinical relevance. The detailed mechanisms that alter this cell growth, morphology and tamoxifen resistance need to be investigated further.

\section{Acknowledgements}

The RAMA 37 and RAMA 37-28 cell lines were the subject of a Material Transfer Agreement between Queen's University Belfast and the University of Ljubljana. This study was supported by a Young Researcher grant to N.T., a J3-0124 grant to N.D., and P1-0390 Research Programme to R.K, all from the Slovenian Research Agency (ARRS). This study was also supported by a VEGA 1/0394/15 grant to P.S. from the Scientific Grant Agency of the Ministry of Education, Science, Research and Sport of the Slovak Republic, and internal scientific grants VVGS-2014190 to L.I. and VVGS-PF-2016-72617 to E.S. from Pavol Jozef Šafárik University, Slovakia. The authors thank Dr Chris Berrie for critical appraisal and editing of the manuscript.

\section{References}

1. Bazan JF: Haemopoietic receptors and helical cytokines Immunol Today 11: 350-354, 1990.

2. Elliott $S$ and Sinclair AM: The effect of erythropoietin on normal and neoplastic cells. Biologics 6: 163-189, 2012.

3. Fraser M, Bai T and Tsang BK: Akt promotes cisplatin resistance in human ovarian cancer cells through inhibition of p53 phosphorylation and nuclear function. Int J Cancer 122: 534-546, 2008.

4. Richmond TD, Chohan M and Barber DL: Turning cells red: Signal transduction mediated by erythropoietin. Trends Cell Biol 15: 146-155, 2005.

5. Koury MJ and Bondurant MC: The molecular mechanism of erythropoietin action. Eur J Biochem 210: 649-663, 1992.

6. Masuda S, Nagao M, Takahata K, Konishi Y, Gallyas F Jr, Tabira T and Sasaki R: Functional erythropoietin receptor of the cells with neural characteristics. Comparison with receptor properties of erythroid cells. J Biol Chem 268: 11208-11216, 1993.

7. Anagnostou A, Liu Z, Steiner M, Chin K, Lee ES, Kessimian N and Noguchi CT: Erythropoietin receptor mRNA expression in human endothelial cells. Proc Natl Acad Sci USA 91: 3974-3978, 1994.

8. Westenfelder C, Biddle DL and Baranowski RL: Human, rat, and mouse kidney cells express functional erythropoietin receptors. Kidney Int 55: 808-820, 1999.

9. Ogilvie M, Yu X, Nicolas-Metral V, Pulido SM, Liu C, Ruegg UT and Noguchi CT: Erythropoietin stimulates proliferation and interferes with differentiation of myoblasts. J Biol Chem 275: 39754-39761, 2000.

10. Abhold E, Rahimy E, Wang-Rodriguez J, Blair KJ, Yu MA, Brumund KT, Weisman RA and Ongkeko WM: Recombinant human erythropoietin promotes the acquisition of a malignant phenotype in head and neck squamous cell carcinoma cell lines in vitro. BMC Res Notes 4: 553, 2011.

11. Lopez TV, Lappin TR, Maxwell P, Shi Z, Lopez-Marure R, Aguilar C and Rocha-Zavaleta L: Autocrine/paracrine erythropoietin signalling promotes JAK/STAT-dependent proliferation of human cervical cancer cells. Int J Cancer 129: 2566-2576, 2011.

12. Pérès EA, Valable $S$, Guillamo JS, Marteau L, Bernaudin JF, Roussel S, Lechapt-Zalcman E, Bernaudin M and Petit E: Targeting the erythropoietin receptor on glioma cells reduces tumour growth. Exp Cell Res 317: 2321-2332, 2011.

13. Wu P, Zhang N, Wang X, Zhang C, Li T, Ning X and Gong K: The erythropoietin/erythropoietin receptor signaling pathway promotes growth and invasion abilities in human renal carcinoma cells. PLoS One 7: e45122, 2012.

14. Zhou B, Damrauer JS, Bailey ST, Hadzic T, Jeong Y, Clark K, Fan C, Murphy L, Lee CY, Troester MA, et al: Erythropoietin promotes breast tumorigenesis through tumor-initiating cell selfrenewal. J Clin Invest 124: 553-563, 2014.
15. Arcasoy MO: Non-erythroid effects of erythropoietin. Haematologica 95: 1803-1805, 2010.

16. Debeljak N, Solár P and Sytkowski AJ: Erythropoietin and cancer: The unintended consequences of anemia correction. Front Immunol 5: 563, 2014.

17. Schnitt SJ: Classification and prognosis of invasive breast cancer: From morphology to molecular taxonomy. Mod Pathol 23 (Suppl 2): S60-S64, 2010.

18. Anderson WF, Chatterjee N, Ershler WB and Brawley OW: Estrogen receptor breast cancer phenotypes in the Surveillance, Epidemiology, and End Results database. Breast Cancer Res Treat 76: 27-36, 2002.

19. Ali S and Coombes RC: Endocrine-responsive breast cancer and strategies for combating resistance. Nat Rev Cancer 2: 101-112, 2002.

20. Jensen EV and Jordan VC: The estrogen receptor: A model for molecular medicine. Clin Cancer Res 9: 1980-1989, 2003.

21. Jordan VC and Koerner S: Tamoxifen (ICI 46,474) and the human carcinoma $8 \mathrm{~S}$ oestrogen receptor. Eur J Cancer 11: 205-206, 1975.

22. Chen H, Tritton TR, Kenny N, Absher M and Chiu JF: Tamoxifen induces TGF-beta 1 activity and apoptosis of human MCF-7 breast cancer cells in vitro. J Cell Biochem 61: 9-17, 1996.

23. Ellis PA, Saccani-Jotti G, Clarke R, Johnston SR, Anderson E, Howell A, A'Hern R, Salter J, Detre S, Nicholson R, et al: Induction of apoptosis by tamoxifen and ICI 182780 in primary breast cancer. Int J Cancer 72: 608-613, 1997.

24. Mabuchi S, Ohmichi M, Kimura A, Ikebuchi Y, Hisamoto K, Arimoto-Ishida E, Nishio Y, Takahashi K, Tasaka K and Murata Y: Tamoxifen inhibits cell proliferation via mitogenactivated protein kinase cascades in human ovarian cancer cell lines in a manner not dependent on the expression of estrogen receptor or the sensitivity to cisplatin. Endocrinology 145: 1302-1313, 2004.

25. Jordan VC: Tamoxifen (ICI46,474) as a targeted therapy to treat and prevent breast cancer. Br J Pharmacol 147 (Suppl 1): S269-S276, 2006.

26. Hopp TA and Fuqua SA: Estrogen receptor variants. J Mammary Gland Biol Neoplasia 3: 73-83, 1998.

27. Milani A, Geuna E, Mittica G and Valabrega G: Overcoming endocrine resistance in metastatic breast cancer: Current evidence and future directions. World J Clin Oncol 5: 990-1001, 2014.

28. Viedma-Rodríguez R, Baiza-Gutman L, Salamanca-Gómez F, Diaz-Zaragoza M, Martínez-Hernández G, Ruiz EsparzaGarrido R, Velázquez-Flores MA and Arenas-Aranda D: Mechanisms associated with resistance to tamoxifen in estrogen receptor-positive breast cancer (Review). Oncol Rep 32: 3-15, 2014.

29. Clark AS, West K, Streicher S and Dennis PA: Constitutive and inducible Akt activity promotes resistance to chemotherapy, trastuzumab, or tamoxifen in breast cancer cells. Mol Cancer Ther 1: 707-717, 2002.

30. Larsson AM, Jirström K, Fredlund E, Nilsson S, Rydén L, Landberg G and Påhlman S: Erythropoietin receptor expression and correlation to tamoxifen response and prognosis in breast cancer. Clin Cancer Res 15: 5552-5559, 2009.

31. Van de Sompele J, De Preter K, Pattyn F, Poppe B, Van Roy N, De Paepe A and Speleman F: Accurate normalization of real-time quantitative RT-PCR data by geometric averaging of multiple internal control genes. Genome Biol 3: Research0034, 2002.

32. Volgger B, Kurz K, Zöschg K, Theurl I, Ciresa-König A, Marth C and Weiss G: Importance of erythropoetin receptor expression in tumour tissue for the clinical course of breast cancer. Anticancer Res 30: 3721-3726, 2010.

33. Trošt N, Hevir N, Rižner TL and Debeljak N: Correlation between erythropoietin receptor(s) and estrogen and progesterone receptor expression in different breast cancer cell lines. Int J Mol Med 31: 717-725, 2013.

34. Reinbothe S, Larsson AM, Vaapil M, Wigerup C, Sun J, Jögi A, Neumann D, Rönnstrand L and Påhlman S: EPO-independent functional EPO receptor in breast cancer enhances estrogen receptor activity and promotes cell proliferation. Biochem Biophys Res Commun 445: 163-169, 2014.

35. Shi Z, Hodges VM, Dunlop EA, Percy MJ, Maxwell AP, El-Tanani M and Lappin TR: Erythropoietin-induced activation of the JAK2/STAT5, PI3K/Akt, and Ras/ERK pathways promotes malignant cell behavior in a modified breast cancer cell line. Mol Cancer Res 8: 615-626, 2010. 
36. Bustin SA, Benes V, Garson JA, Hellemans J, Huggett J, Kubista M, Mueller R, Nolan T, Pfaffl MW, Shipley GL, et al: The MIQE guidelines: Minimum information for publication of quantitative real-time PCR experiments. Clin Chem 55: 611-622, 2009.

37. Simoncini T, Genazzani AR and De Caterina R: Towards a molecular understanding of the atheroprotective effects of estrogens: A review of estrogen effects on endothelial activation. Ital Heart J 1: 104-107, 2000

38. Kutuk O, Arisan ED, Tezil T, Shoshan MC and Basaga H: Cisplatin overcomes Bcl-2-mediated resistance to apoptosis via preferential engagement of Bak: Critical role of Noxa-mediated lipid peroxidation. Carcinogenesis 30: 1517-1527, 2009.

39. Paragh G, Kumar SM, Rakosy Z, Choi SC, Xu X and Acs G: RNA interference-mediated inhibition of erythropoietin receptor expression suppresses tumor growth and invasiveness in A2780 human ovarian carcinoma cells. Am J Pathol 174: 1504-1514, 2009.
40. McGuire WL: Current status of estrogen receptors in human breast cancer. Cancer 36: 638-644, 1975.

41. Michalides R, Griekspoor A, Balkenende A, Verwoerd D, Janssen L, Jalink K, Floore A, Velds A, van't Veer L and Neefjes J: Tamoxifen resistance by a conformational arrest of the estrogen receptor alpha after PKA activation in breast cancer. Cancer Cell 5: 597-605, 2004.

42. Esslimani-Sahla M, Simony-Lafontaine J, Kramar A, Lavaill R, Mollevi C, Warner M, Gustafsson JA and Rochefort H: Estrogen receptor beta (ER beta) level but not its ER beta cx variant helps to predict tamoxifen resistance in breast cancer. Clin Cancer Res 10: 5769-5776, 2004.

43. Campbell RA, Bhat-Nakshatri P, Patel NM, Constantinidou D, Ali S and Nakshatri H: Phosphatidylinositol 3-kinase/AKTmediated activation of estrogen receptor alpha: A new model for anti-estrogen resistance. J Biol Chem 276: 9817-9824, 2001. 